\title{
Size effects in molecular dynamics thermal conductivity predictions
}

\author{
D. P. Sellan, ${ }^{1}$ E. S. Landry, ${ }^{2}$ J. E. Turney, ${ }^{2}$ A. J. H. McGaughey, ${ }^{2, *}$ and C. H. Amon ${ }^{1,2}$ \\ ${ }^{1}$ Department of Mechanical \& Industrial Engineering, University of Toronto, Toronto, Ontario, Canada M5S $3 G 8$ \\ ${ }^{2}$ Department of Mechanical Engineering, Carnegie Mellon University, Pittsburgh, Pennsylvania 15213, USA
}

(Received 23 March 2010; revised manuscript received 3 June 2010; published 21 June 2010)

\begin{abstract}
We predict the bulk thermal conductivity of Lennard-Jones argon and Stillinger-Weber silicon using the Green-Kubo (GK) and direct methods in classical molecular dynamics simulations. While system-sizeindependent thermal conductivities can be obtained with less than 1000 atoms for both materials using the GK method, the linear extrapolation procedure [Schelling et al., Phys. Rev. B 65, 144306 (2002)] must be applied to direct method results for multiple system sizes. We find that applying the linear extrapolation procedure in a manner consistent with previous researchers can lead to an underprediction of the GK thermal conductivity (e.g., by a factor of 2.5 for Stillinger-Weber silicon at a temperature of $500 \mathrm{~K}$ ). To understand this discrepancy, we perform lattice dynamics calculations to predict phonon properties and from these, length-dependent thermal conductivities. From these results, we find that the linear extrapolation procedure is only accurate when the minimum system size used in the direct method simulations is comparable to the largest mean-free paths of the phonons that dominate the thermal transport. This condition has not typically been satisfied in previous works. To aid in future studies, we present a simple metric for determining if the system sizes used in direct method simulations are sufficiently large so that the linear extrapolation procedure can accurately predict the bulk thermal conductivity.
\end{abstract}

DOI: 10.1103/PhysRevB.81.214305

PACS number(s): 63.20.kg, 66.70.-f, 05.60.Cd

\section{INTRODUCTION}

The two most common approaches for predicting phonon (i.e., lattice) thermal conductivity, $k$, using molecular dynamics (MD) simulation are the Green-Kubo (GK) method ${ }^{1-15}$ and the direct method. ${ }^{5-8,10,16-22}$ The Green-Kubo method is an equilibrium technique based on the fluctuation-dissipation theorem. Cubic simulation cells with periodic boundary conditions are typically used and size-independent thermal conductivities can be obtained for most materials using fewer than 10000 atoms. Interpreting the results of the GreenKubo method can be challenging for high thermal conductivity materials and systems with complex unit cells, such as superlattices. ${ }^{6,10,11}$ The direct method is a nonequilibrium steady-state technique in which a heat flux is applied to a simulation cell along the direction of interest. Using the imposed heat flux and the resulting steady-state temperature gradient, the Fourier law is applied to calculate the thermal conductivity. For many materials, it is computationally prohibitive to obtain sample size-independent thermal conductivity predictions using the direct method. For such cases, it becomes necessary to make thermal conductivity predictions for several sample lengths and then perform a postprocessing extrapolation procedure.

The extrapolation procedure commonly used with the direct method can be derived using the kinetic theory expression for thermal conductivity and the Matthiessen rule, ${ }^{5}$ resulting in a predicted linear dependence of $1 / k$ on $1 / L$, where $L$ is the sample length. Thermal conductivities for multiple sample lengths are plotted as $1 / k$ versus $1 / L$ and a linear fit to the data is extrapolated to the $1 / L \rightarrow 0$ (i.e., bulk) limit. Using classical MD simulation and empirical interatomic potentials, a linear relationship between $1 / k$ and $1 / L$ has been observed for bulk silicon, ${ }^{5}$ argon, ${ }^{7}$ SiGe alloys, ${ }^{10}$ and diamond, ${ }^{18}$ Lennard-Jones (LJ) (Ref. 6) and Si/Ge (Ref.
10) superlattices, and silicon ${ }^{19,20}$ and $\mathrm{SiC}$ (Ref. 21) nanowires. Studying gallium nitride $(\mathrm{GaN})$ samples up to $128 \mathrm{~nm}$ in length, Zhou et al. ${ }^{8}$ found that a linear relationship reasonably captures the $1 / k$ dependence on $1 / L$ at a temperature of $300 \mathrm{~K}$. At a temperature of $800 \mathrm{~K}$, however, they found a nonlinear dependence. For carbon nanotubes, Thomas et al. ${ }^{22}$ obtained sample size-independent thermal conductivity predictions for tube lengths on the order of $1 \mu \mathrm{m}$. A nonlinear relationship is observed when their $1 / k$ data is plotted versus $1 / L$.

Studying bulk LJ argon ${ }^{7}$ and LJ superlattices, ${ }^{6}$ Landry, McGaughey and co-workers found agreement between Green-Kubo and direct method thermal conductivities to within the prediction uncertainties for temperatures between 20 and $80 \mathrm{~K}$ [the melting temperature of LJ argon is $\sim 87 \mathrm{~K}$ (Ref. 23)]. For Stillinger-Weber (SW) silicon, Schelling et $a l .{ }^{5}$ found agreement between the two methods at a temperature of $1000 \mathrm{~K}$. In Sec. II, however, we will show that a seemingly accurate application of the direct method extrapolation procedure to SW silicon at a temperature of $500 \mathrm{~K}$ results in an underprediction of the Green-Kubo thermal conductivity by a factor of 2.5 . This result, along with the nonlinear results of Zhou et al. ${ }^{8}$ and Thomas et al., ${ }^{22}$ call into question the general validity of the linear extrapolation procedure.

In Sec. III, we assess the validity of the linear extrapolation procedure by considering two systems: (i) LJ argon, where the two methods are in agreement for all temperatures and (ii) SW silicon, where the two methods are in better agreement at higher temperatures. To do so, sample lengths that generate length-independent thermal conductivities (tens of micron for SW silicon) must be accessed. To predict the thermal conductivity of such systems using the direct method is computationally prohibitive. To overcome this challenge, we use lattice dynamics calculations to predict phonon properties and thermal conductivities. This ability allows us to 
resolve nonlinearities in the $1 / k$ versus $1 / L$ trend that only become apparent for SW silicon samples longer than a few microns. We use these results to identify when the linear extrapolation procedure will accurately predict the bulk thermal conductivity.

In Sec. IV, we develop an analytical model for the length dependence of thermal conductivity. We then use this model to derive a metric that can be used to assess whether the sample sizes used in a direct method MD simulation are sufficiently large to accurately predict the bulk thermal conductivity using the linear extrapolation procedure.

\section{PREDICTING THERMAL CONDUCTIVITY USING MOLECULAR DYNAMICS SIMULATION}

\section{A. Green-Kubo method}

In the Green-Kubo method, the thermal conductivity, $k_{\mathrm{GK}}$, is predicted using the equilibrium fluctuations of the heat current vector, $\mathbf{S}$, via the fluctuation-dissipation theorem. For a cubically isotropic material, the diagonal components of the thermal conductivity tensor can be averaged to give ${ }^{2}$

$$
k_{\mathrm{GK}}=\frac{1}{3 k_{\mathrm{B}} V T^{2}} \int_{0}^{\infty}\langle\mathbf{S}(t) \cdot \mathbf{S}(0)\rangle d t,
$$

where $k_{\mathrm{B}}$ is the Boltzmann constant, $V$ and $T$ are the system volume and absolute temperature, $t$ is the time, and $\langle\mathbf{S}(t) \cdot \mathbf{S}(0)\rangle$ is the heat current autocorrelation function (HCACF). The details of our Green-Kubo methodology can be found in Refs. 9 and 11.

Two challenges are encountered when applying the Green-Kubo method to predict thermal conductivity. The first challenge is to accurately specify the converged value of the HCACF integral, which is proportional to the thermal conductivity through Eq. (1). The HCACFs (normalized by their initial values) and their integrals for SW silicon at temperatures of 500 and $1000 \mathrm{~K}$ are shown in Fig. 1. For both cases, the simulation cells contain $6^{3}$ conventional diamond unit cells $(N=1728$ atoms $)$ and the data correspond to the average of ten independent simulations of $1600 \mathrm{ps}\left(3 \times 10^{6}\right.$ time steps). While the HCACF integral has converged after $400 \mathrm{ps}$ for both temperatures, it begins to drift after $800 \mathrm{ps}$ due to noise in the HCACF. ${ }^{5}$ For SW silicon, we specify the converged value of the HCACF integral by averaging its value between times of 400 and 800 ps (the shaded region of the inset in Fig. 1). Specifying the converged value of the HCACF integral for LJ argon, where the HCACF has less noise, ${ }^{7}$ is less challenging.

To ensure that sufficient data are available to accurately predict the converged value of the HCACF integral, the total simulation time should be many times greater than the largest phonon relaxation times that dominate the thermal conductivity. For this reason, it can be challenging to apply the Green-Kubo method for high thermal conductivity materials, which typically have large relaxation times. For some complex unit-cell materials (e.g., superlattices), the amount of noise in the HCACF makes it impossible to specify a converged HCACF integral, even after increasing the total simulation time and averaging over independent simulations. ${ }^{6,10,11}$

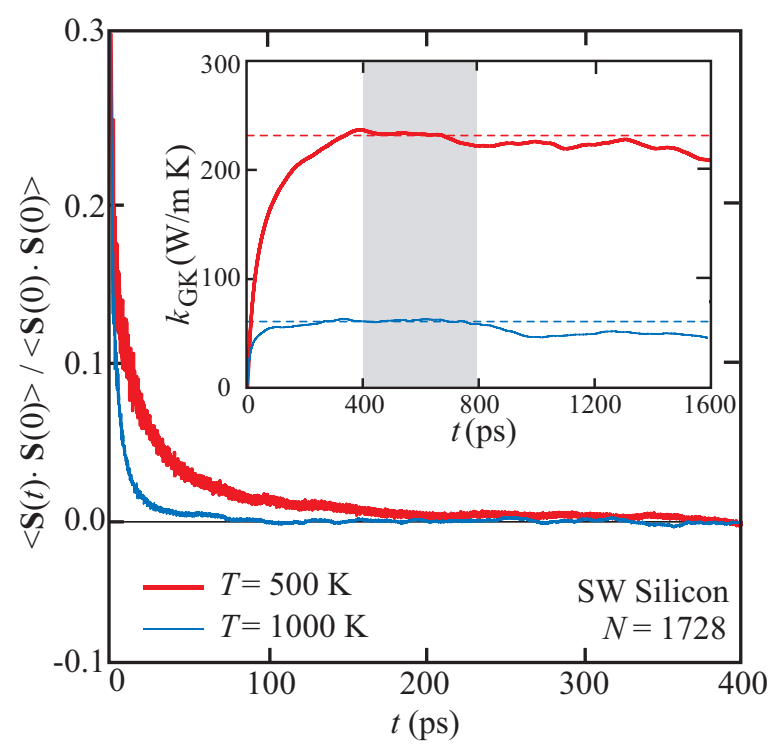

FIG. 1. (Color online) Heat current autocorrelation function (body) and its integral (inset) for SW silicon at temperatures of 500 and $1000 \mathrm{~K}$. The HCACFs are normalized by their initial values. The shaded region in the inset indicates the time range over which the HCACF integral is averaged to predict the thermal conductivity, $k_{\mathrm{GK}}$, which is represented by a dashed line for each temperature. Note that the time scale of the inset is four times that of the body.

For some one-dimensional lattices (e.g., molecular chains), the HCACF has been observed to diverge. ${ }^{12-14}$

The second challenge is to address the effect of the finite simulation cell size. The thermal conductivity will depend on the size of the simulation cell if there are not enough phonon modes to accurately reproduce the phonon-phonon scattering in the associated bulk material [i.e., the Brillouin-zone (BZ) resolution is too coarse]. This effect can be removed by increasing the simulation cell size until the thermal conductivity reaches a size-independent value. The effect of the simulation cell size on the thermal conductivity of bulk SW silicon at a temperature of $500 \mathrm{~K}$ is provided in Table I. The prediction uncertainties are the $95 \%$ confidence intervals based on the results of ten independent simulations of 1600 ps. The uncertainty is larger for SW silicon $(\sim 20 \%)$ than for LJ argon $(\sim 5 \%) .9$ There is no discernible size dependence for simulation cells containing $4^{3}$ to $8^{3}$ conventional unit cells, a finding that is in agreement with the results of Schell-

TABLE I. Size dependence of SW silicon thermal conductivity at a temperature of $500 \mathrm{~K}$ predicted using MD simulations and the Green-Kubo method. The prediction uncertainty is the $95 \%$ confidence interval based on the results of ten independent simulations.

\begin{tabular}{lcc}
\hline \hline $\begin{array}{l}\text { Cell size } \\
\text { (conventional unit cells) }\end{array}$ & $\begin{array}{c}\text { Number of atoms, } \\
N\end{array}$ & $\begin{array}{c}k_{\mathrm{GK}} \\
(\mathrm{W} / \mathrm{m} \mathrm{K})\end{array}$ \\
\hline $4^{3}$ & 512 & $233 \pm 45$ \\
$5^{3}$ & 1000 & $283 \pm 59$ \\
$6^{3}$ & 1728 & $230 \pm 37$ \\
$7^{3}$ & 2744 & $181 \pm 47$ \\
$8^{3}$ & 4096 & $231 \pm 57$ \\
\hline \hline
\end{tabular}


TABLE II. Bulk thermal conductivities, in W/m K, for SW silicon and LJ argon found using the GreenKubo method $\left(k_{\mathrm{GK}}\right.$, Sec. II A) and the direct method $\left(k_{\mathrm{DM}}^{e}\right.$, Sec. II B) in MD simulations and from lattice dynamics calculations $\left(k_{\mathrm{LD}}\right.$ and $k_{\mathrm{LD}}^{e}$, Sec. III). The direct method uncertainty is estimated to be $\pm 20 \%$ for SW silicon and $\pm 10 \%$ for LJ Argon based on the prediction repeatability. The superscript $e$ indicates that the value was predicted using the linear extrapolation procedure.

\begin{tabular}{lccccc}
\hline \hline & \multicolumn{2}{c}{ Molecular dynamics } & & \multicolumn{2}{c}{ Lattice dynamics } \\
\cline { 2 - 3 } \cline { 5 - 6 } System & $k_{\mathrm{GK}}$ & $k_{\mathrm{DM}}^{e}$ & & $k_{\mathrm{LD}}$ & $k_{\mathrm{LD}}^{e}$ \\
\hline SW silicon, $T=500 \mathrm{~K}$ & $231 \pm 57$ & $93 \pm 18$ & & $275^{\mathrm{a}}$ & 132 \\
$\mathrm{SW}$ silicon, $T=1000 \mathrm{~K}$ & $60 \pm 12$ & $40 \pm 8$ & & $122^{\mathrm{a}}$ & 69 \\
$\mathrm{LJ}$ argon, $T=40 \mathrm{~K}$ & $0.47 \pm 0.02^{\mathrm{b}}$ & $0.50 \pm 0.05^{\mathrm{b}}$ & & $0.62^{\mathrm{b}}$ & 0.61 \\
\hline \hline
\end{tabular}

${ }^{\mathrm{a}}$ Reference 24.

${ }^{b}$ Reference 7.

ing et al. ${ }^{5}$ The thermal conductivity predicted using $8^{3}$ conventional unit cells will be used when comparing to the direct method predictions for SW silicon at a temperature of $500 \mathrm{~K}$. To reduce computational load, $6^{3}$ conventional unit cells are used to generate the other Green-Kubo predictions.

The Green-Kubo predicted thermal conductivities for SW silicon and LJ argon (at a temperature of $40 \mathrm{~K}$ ) are provided in Table II. The SW silicon prediction at a temperature of $1000 \mathrm{~K}$ is in agreement with the predictions of Schelling et $a l .{ }^{5}$ and Goicochea et $a l . .^{25}$

\section{B. Direct method}

\section{Overview}

In the direct method, a known heat flux, $q$, is applied across a sample of finite length and the resulting temperature gradient, $\partial T / \partial z$, is calculated. The thermal conductivity, $k_{\mathrm{DM}}$, is then determined using the Fourier law,

$$
k_{\mathrm{DM}}=-\frac{q}{\partial T / \partial z} .
$$

The simulation cell configuration used in our direct method simulations is shown in Fig. 2. The simulation cell consists of a sample region of length $L$ bordered by hot and cold reservoirs in the $z$ direction. Periodic boundary conditions are applied in the $x$ and $y$ directions. Fixed boundaries bound the reservoirs to prevent the sublimation of reservoir atoms. Details related to our direct method methodology are available elsewhere. ${ }^{7,11}$

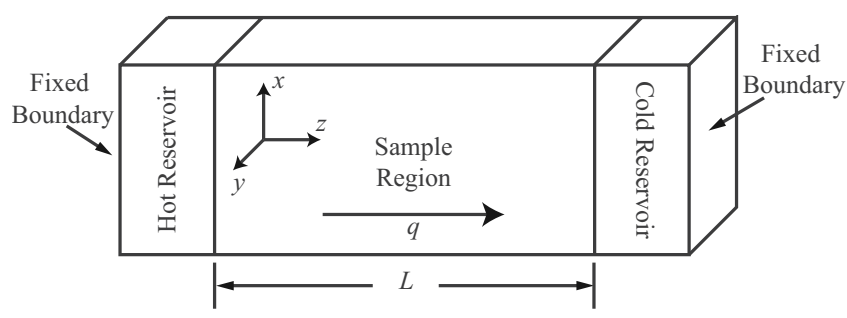

FIG. 2. Schematic diagram of the simulation cell configuration used in the direct method simulations.

\section{Simulation cell size effects}

The size dependence of the thermal conductivity predicted in a direct method simulation can arise in two ways. The first way is the same phenomenon that occurs in the Green-Kubo method. For samples that are too small, the BZ resolution is too coarse and too few phonon modes are available to accurately reproduce the phonon-phonon scattering present in the bulk material. Because direct method simulation cells are much larger than those used for Green-Kubo simulations, this effect is negligible.

The second mechanism by which size dependence arises is through phonon scattering at the sample/reservoir boundaries. This effect is significant when the sample length is smaller than the bulk phonon mean-free paths, $\Lambda_{\infty}(\boldsymbol{\kappa}, \nu)$ (here, the subscript $\infty$ refers to bulk and each phonon mode is identified by its wave vector, $\boldsymbol{\kappa}$, and dispersion branch, $\nu$ ). If the sample length is smaller than $\Lambda_{\infty}(\boldsymbol{\kappa}, \nu)$, a phonon of mode $(\boldsymbol{\kappa}, \nu)$ emitted from the hot reservoir can travel across the sample without scattering (i.e., ballistic transport). As the sample length is increased, phonons will have a greater likelihood of scattering before they reach the other sample/ reservoir boundary, resulting in a transition to diffusive (i.e., bulklike) transport. Phonons traveling ballistically contribute less to the thermal conductivity than in bulk as their meanfree paths are reduced to the sample length. Thermal conductivity is therefore a length-dependent property when ballistic effects are present.

To describe this length dependence, we now present a model based the Boltzmann transport equation (BTE) and the Matthiessen rule. The derivation begins with an expression for the thermal conductivity in the $z$ direction that is obtained by solving the BTE under the relaxation-time approximation and using the Fourier law ${ }^{26,27}$

$$
k(L)=\sum_{\nu} \sum_{\boldsymbol{\kappa}} c_{p h} v_{g, z}^{2}(\boldsymbol{\kappa}, \nu) \tau(\boldsymbol{\kappa}, \nu, L) .
$$

The summation is over all phonon modes, the volumetric specific heat of each mode, $c_{p h}$, is $k_{\mathrm{B}} / V$ for the classical systems considered here, and $v_{g, z}(\boldsymbol{\kappa}, \nu)$ is the $z$ component of the group-velocity vector, $\mathbf{v}_{g}(\boldsymbol{\kappa}, \nu)$. The phonon transport is described using a set of mode- and system-size-dependent relaxation times, $\tau(\boldsymbol{\kappa}, \nu, L)$, defined as the average time be- 
tween successive scattering events, i.e., $\quad \tau(\boldsymbol{\kappa}, \nu, L)$ $=\Lambda(\boldsymbol{\kappa}, \nu, L) /\left|\mathbf{v}_{g}(\boldsymbol{\kappa}, \nu)\right|$.

We apply the Matthiesen rule, which assumes that different scattering mechanisms are independent, to model the length dependence of $\tau(\boldsymbol{\kappa}, \nu, L)$ such that

$$
\frac{1}{\tau(\boldsymbol{\kappa}, \nu, L)}=\frac{1}{\tau_{\infty}(\boldsymbol{\kappa}, \nu)}+\frac{1}{\tau_{b}(\boldsymbol{\kappa}, \nu, L)},
$$

where $\tau_{\infty}(\boldsymbol{\kappa}, \nu)$ and $\tau_{b}(\boldsymbol{\kappa}, \nu, L)$ are the intrinsic scattering and boundary scattering relaxation times. We assume that the crystal contains no defects, no free electrons, and no internal interfaces so that $\tau_{\infty}(\boldsymbol{\kappa}, \nu)$ is equal to the relaxation time associated with phonon-phonon scattering. The boundary scattering relaxation time is taken to be the average time between boundary scattering events in the absence of intrinsic scattering, i.e.,

$$
\tau_{b}(\boldsymbol{\kappa}, \nu, L)=\frac{L / 2}{\left|v_{g, z}(\boldsymbol{\kappa}, \nu)\right|} .
$$

Substituting Eq. (4) into Eq. (3) and applying Eq. (5) leads to an expression that describes the length dependence of thermal conductivity,

$$
\begin{aligned}
k(L)= & \sum_{\nu} \sum_{\boldsymbol{\kappa}} c_{p h} v_{g, z}^{2}(\boldsymbol{\kappa}, \nu) \tau_{\infty}(\boldsymbol{\kappa}, \nu) \\
& \times\left[1+\frac{2\left|v_{g, z}(\boldsymbol{\kappa}, \nu)\right| \tau_{\infty}(\boldsymbol{\kappa}, \nu)}{L}\right]^{-1} .
\end{aligned}
$$

As $L$ approaches infinity, the bracketed term in Eq. (6) approaches unity and $k_{z}(L)$ approaches the bulk value, $k_{\infty}$.

\section{Motivating the linear extrapolation procedure}

Motivated by Eq. (6), we can describe the length dependence of thermal conductivity using $1 / k$ as a function of $1 / L$, i.e.,

$$
\frac{1}{k}=\mathcal{X}\left(\frac{1}{L}\right) .
$$

Here, $\mathcal{X}$ is an unknown function of $1 / L$ that converges to $1 / k_{\infty}$ as $1 / L \rightarrow 0$. The bulk thermal conductivity can be estimated using $\mathcal{X}$ and its derivatives with respect to $1 / L\left(\mathcal{X}^{\prime}\right.$, $\mathcal{X}^{\prime \prime}$, etc.) at a finite sample length and extrapolating to $\mathcal{X}(0)$ using a Taylor-series expansion, i.e.,

$$
\begin{aligned}
\frac{1}{k_{\infty}}= & \mathcal{X}(0)=\mathcal{X}(1 / L)+\frac{\mathcal{X}^{\prime}(1 / L)}{1 !}\left[-\frac{1}{L}\right]+\frac{\mathcal{X}^{\prime \prime}(1 / L)}{2 !}\left[-\frac{1}{L}\right]^{2} \\
& +\cdots
\end{aligned}
$$

To obtain the derivatives of $\mathcal{X}$, one could use the direct method to predict the thermal conductivities for a range of sample sizes and take the derivatives of a polynomial fit to $1 / k$ (i.e., $\mathcal{X}$ ) versus $1 / L$. It is difficult, however, to accurately predict the second-order and higher-order derivatives with this approach due to uncertainty in the direct method predictions. Additionally, to predict an $N$ th order derivative requires at least $N+1$ sample sizes to be considered, which can be computationally expensive. To avoid these challenges, (a)

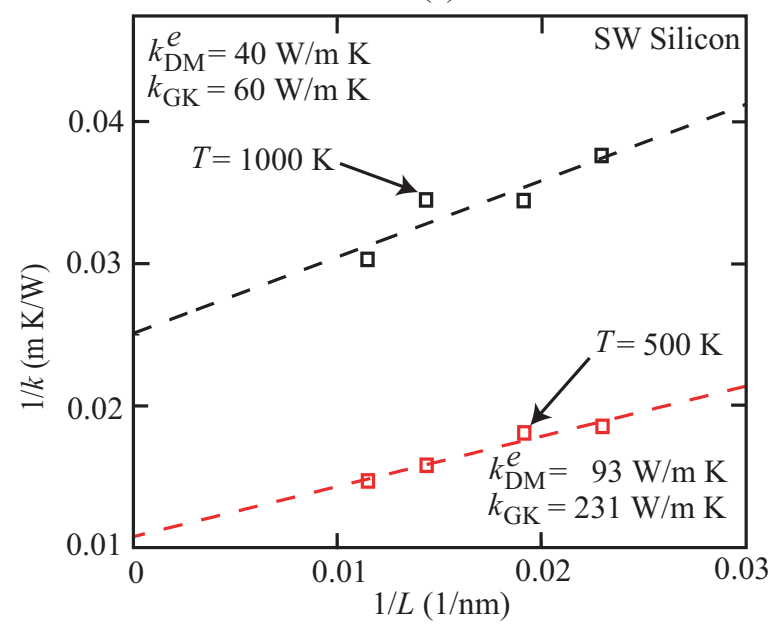

(b)

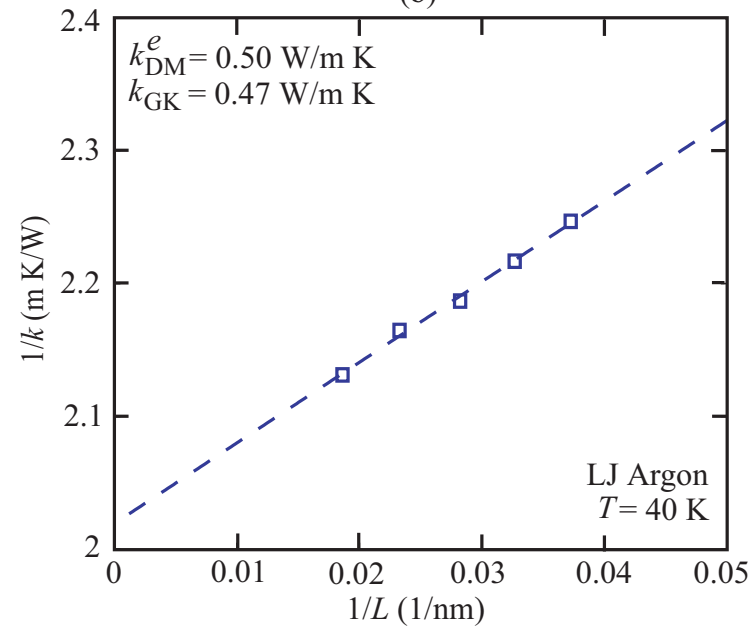

FIG. 3. (Color online) Length-dependent thermal conductivities for (a) SW silicon at temperatures of 500 and $1000 \mathrm{~K}$ and (b) LJ argon at a temperature of $40 \mathrm{~K}$ predicted from the direct method and MD simulations. The dashed lines are linear fits to the discrete data.

$1 / k_{\infty}$ can be approximated by truncating Eq. (8) after the first-order term. Under this first-order approximation, the procedure for estimating the bulk thermal conductivity reduces to plotting $1 / k$ versus $1 / L$ for a range of sample lengths and extrapolating a linear fit to the data to $1 / L=0$. This procedure is equivalent to that proposed by Schelling et $a l .{ }^{5}$ who derived it by assuming that all the phonon modes in Eq. (6) have the same, averaged properties. We will demonstrate the conditions under which the linear extrapolation procedure (i.e., the first-order approximation) is valid in Sec. III B.

\section{Direct method thermal conductivity predictions}

Our direct method thermal conductivity predictions are shown in Figs. 3(a) and 3(b). The linearly extrapolated bulk values, $k_{\mathrm{DM}}^{e}$, are provided in Table II. The discrete data points in Fig. 3(a) represent predictions for SW silicon samples with lengths ranging from 40 to $80 \mathrm{~nm}$. In Fig. 3(b), the results for $\mathrm{LJ}$ argon samples with lengths ranging from 26 to 
$54 \mathrm{~nm}$ are presented. These ranges of sample lengths are typical of previous work. ${ }^{5-8,10,16-22}$ A linear relationship between $1 / k$ and $1 / L$ is observed for both SW silicon and $\mathrm{LJ}$ argon.

For SW silicon, the bulk thermal conductivities extrapolated from the direct method data underpredict the values predicted using the Green-Kubo method at both temperatures (see Table II). The underprediction is more severe at a temperature of $500 \mathrm{~K}\left(k_{\mathrm{DM}}^{e} / k_{\mathrm{GK}}=0.40\right)$ than at a temperature of $1000 \mathrm{~K}\left(k_{\mathrm{DM}}^{e} / k_{\mathrm{GK}}=0.66\right)$. Schelling et al. ${ }^{5}$ report $1000 \mathrm{~K}$ direct method results using larger sample lengths (up to 209 $\mathrm{nm})$ that are in better agreement with their Green-Kubo result $\left(k_{\mathrm{DM}}^{e} / k_{\mathrm{GK}}=0.95\right)$. For LJ argon, the extrapolated bulk thermal conductivity from the direct method data is within the uncertainty of the Green-Kubo prediction. As reported by Turney et al., ${ }^{7}$ the predictions of these two methods are within the prediction uncertainties for temperatures ranging from 20 to $80 \mathrm{~K}$. To understand why the Green-Kubo and direct method results are in better agreement for LJ argon than for SW silicon and why the effect is temperature dependent for SW silicon, we next present a carrier-level analysis that uses Eq. (6) and phonon properties predicted from lattice dynamics calculations.

\section{PREDICTING THERMAL CONDUCTIVITY USING LATTICE DYNAMICS CALCULATIONS}

\section{A. Phonon properties}

The advantage of using Eq. (6) to predict thermal conductivity is that length scales that are computationally prohibitive with direct method MD simulations can be studied. To predict the phonon properties required to evaluate Eq. (6), we use harmonic and anharmonic lattice dynamics calculations. Harmonic lattice dynamics calculations use the second-order derivatives of the interatomic potential to calculate the phonon frequencies $[\omega(\boldsymbol{\kappa}, \nu)]$. In an anharmonic lattice dynamics calculation, the harmonic solution is perturbed by the third-order and fourth-order derivatives of the interatomic potential, providing the anharmonic shifts in the phonon frequencies and the phonon-phonon relaxation times. The phonon group velocities are found from $\partial \omega / \partial \kappa$. The phonon properties predicted from lattice dynamics calculations can depend on the resolution of the BZ. To eliminate this size effect, the BZ resolution is increased until sizeindependent phonon properties and bulk thermal conductivity are attained. To meet these criteria, we use the conventional unit cell and a grid of $12 \times 12 \times 12$ wave vectors for both LJ argon and SW silicon. A description of lattice dynamics calculations can be found elsewhere. , 28,29 $^{2}$

The bulk thermal conductivities calculated using the lattice dynamics-predicted phonon properties are presented in Table II as $k_{\mathrm{LD}}$. Because the lattice dynamics techniques do not include the full anharmonicity of the atomic interactions (i.e., they neglect the fifth-order and higher-order derivatives of the interatomic potential), $k_{\mathrm{LD}}$ is larger than $k_{\mathrm{GK}}$. We further discuss this effect in Sec. III B.

\section{B. Assessing the linear extrapolation procedure}

We predict a length-dependent thermal conductivity, $k_{\mathrm{LD}}(L)$, using Eq. (6) and the phonon properties predicted (a)

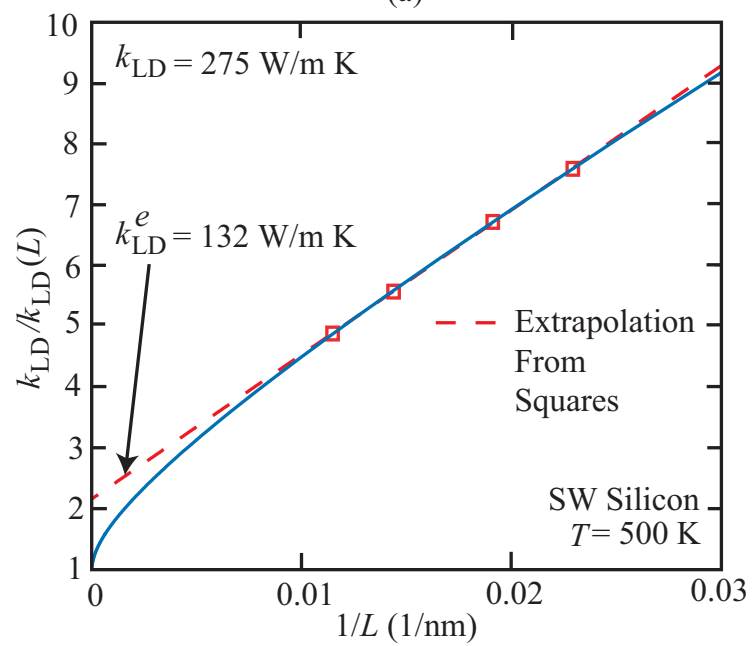

(b)

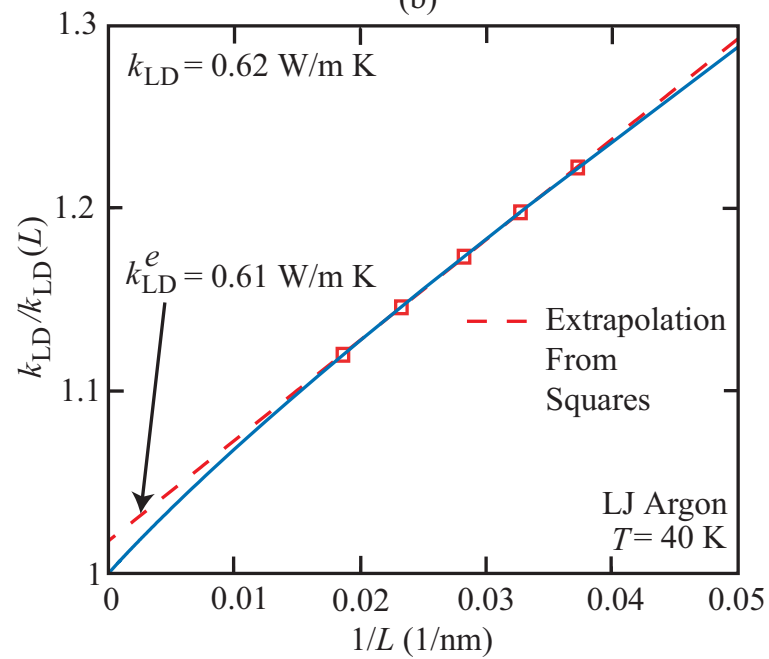

FIG. 4. (Color online) Inverse of the normalized lengthdependent thermal conductivities for (a) SW silicon $(T=500 \mathrm{~K})$ and (b) LJ $\operatorname{argon}(T=40 \mathrm{~K})$. The squares correspond to the sample lengths used in the direct method simulations [see Figs. 3(a) and 3(b)] but are calculated using phonon properties obtained from lattice dynamics calculations. Note the difference in the scales of the vertical axes.

from lattice dynamics calculations. The inverse of the normalized thermal conductivity, $k_{\mathrm{LD}} / k_{\mathrm{LD}}(L)$, is plotted as a solid line in Fig. 4(a) (SW silicon at $T=500 \mathrm{~K}$ ) and Fig. 4(b) (LJ $\operatorname{argon}$ at $T=40 \mathrm{~K}$ ) as a function of the inverse of the sample length, $1 / L$. The discrete data points in Figs. 4(a) and 4(b) correspond to the sample lengths used in the direct method calculations shown in Figs. 3(a) and 3(b) but are calculated using Eq. (6) and phonon properties from lattice dynamics calculations. The dashed line in each of Figs. 4(a) and 4(b) is a linear fit to these discrete data points. Using these linear fits to extrapolate bulk thermal conductivities, $k_{\mathrm{LD}}^{e}$, results in values that are $48 \%, 57 \%$, and $99 \%$ of $k_{\mathrm{LD}}$ for SW silicon at temperatures of 500 and $1000 \mathrm{~K}$ (not shown) and LJ argon at a temperature of $40 \mathrm{~K}$.

As observed with the MD results (see $k_{\mathrm{GK}}$ and $k_{\mathrm{DM}}^{e}$ in Table II), using the linear extrapolation procedure to predict the bulk thermal conductivity yields less error for LJ argon 


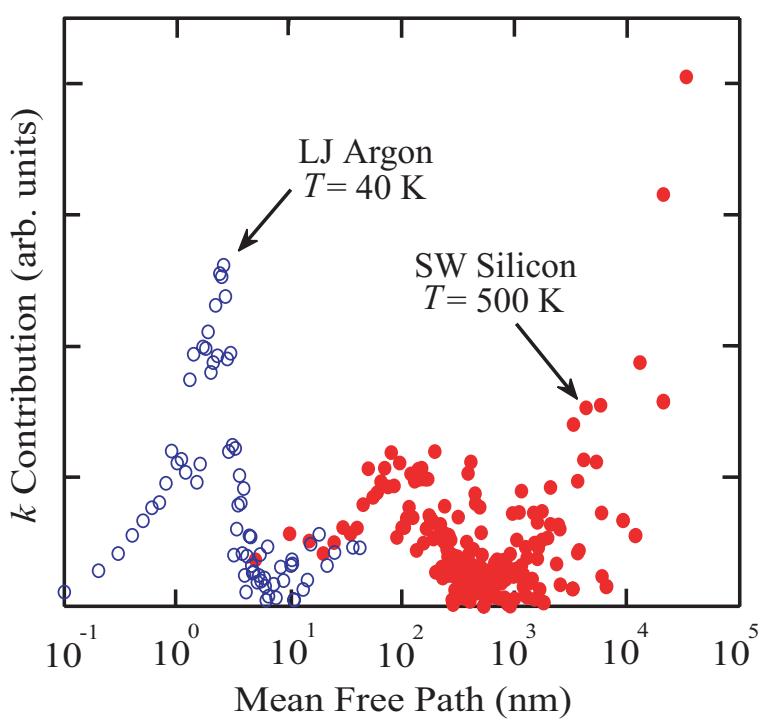

FIG. 5. (Color online) Bulk thermal conductivity contribution dependence on mean-free path for SW silicon $(T=500 \mathrm{~K})$ and $\mathrm{LJ}$ $\arg$ on $(T=40 \mathrm{~K})$. The mean-free paths for each mode are sorted using a histogram with a bin width of $2 \mathrm{~nm}$ for SW silicon and 0.1 $\mathrm{nm}$ for LJ argon.

than for SW silicon. To explain this result, we must assess when neglecting the second-order and higher-order terms in Eq. (8) is valid. Using Eq. (6), it can be shown that $1 / k$ and $1 / L$ are linearly dependent if each phonon property is well approximated by an average value. Since we consider classical systems, the phonon specific heat is equal to $k_{\mathrm{B}} / V$ for all modes. To check if this condition is also true for the group velocities and relaxation times, we plot the bulk thermal conductivity contribution dependence on the phonon mean-free path $\left[\Lambda(\boldsymbol{\kappa}, \nu)=\left|\mathbf{v}_{g}(\boldsymbol{\kappa}, \nu)\right| \tau_{\infty}(\boldsymbol{\kappa}, \nu)\right]$ in Fig. 5. For LJ argon, thermal conductivity is dominated by phonons with meanfree paths that span $0.5-3 \mathrm{~nm}$. This small spread suggests that using an average mean-free path to describe the phonon transport is reasonable. The near linear dependence of $k_{\mathrm{LD}} / k_{\mathrm{LD}}(L)$ on $1 / L$ in Fig. $4(\mathrm{~b})$ and the accuracy of the bulk thermal conductivity predicted using the linear extrapolation procedure are therefore not surprising.

For SW silicon, phonons with mean-free paths that span several orders of magnitude $\left(10^{2}-10^{4} \mathrm{~nm}\right)$ contribute significantly to the bulk thermal conductivity, thus generating the strong deviation from linearity found when $k_{\mathrm{LD}} / k_{\mathrm{LD}}(L)$ is plotted versus $1 / L$ in Fig. 4(a). Using a first-order Taylorseries expansion (i.e., the linear extrapolation procedure) to model such a nonlinear trend is only accurate around the point where the Taylor series is evaluated. Accurately predicting a bulk thermal conductivity thus requires consideration of sample lengths that correspond to the largest bulk mean-free paths that dominate the thermal transport. The largest bulk mean-free paths for SW silicon are two orders of magnitude larger than the sample lengths we considered. This difference makes the linear extrapolation procedure underpredict $k_{\mathrm{LD}}$ by a factor of 2.1 at a temperature of $500 \mathrm{~K}$. When sample lengths comparable to the largest bulk meanfree paths are considered, the error associated with the firstorder Taylor-series approximation decreases. This trend is

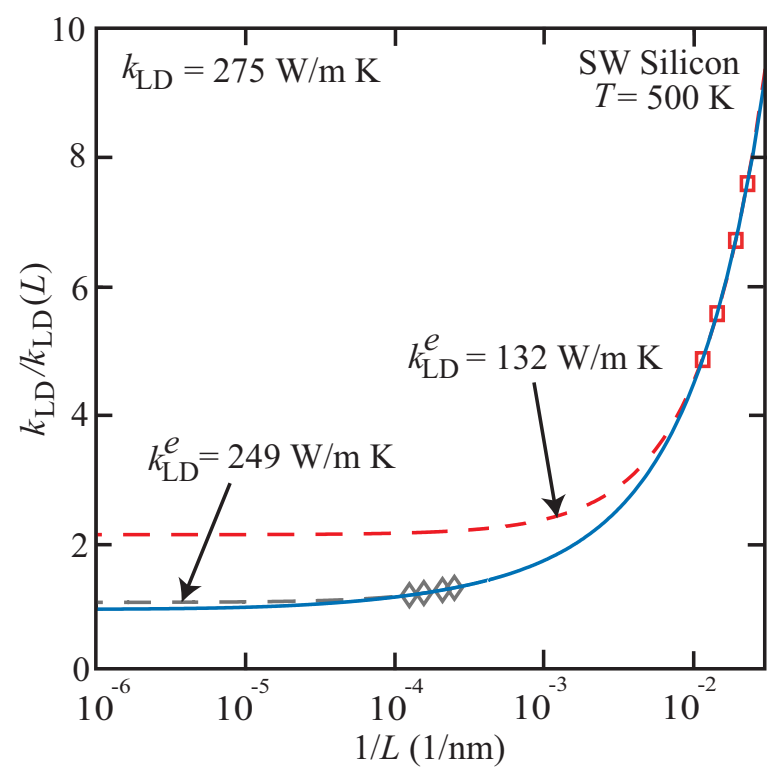

FIG. 6. (Color online) Inverse of the normalized lengthdependent thermal conductivity for SW silicon at $T=500 \mathrm{~K}$ (solid line). The squares correspond to the data in Fig. 4(a) [note that this figure has a logarithmic horizontal axis]. The diamonds correspond to sample lengths between 4 and $8 \mu \mathrm{m}$.

seen in Fig. 6, where thermal conductivities predicted using sample lengths between 4 and $8 \mu \mathrm{m}$ are plotted and the linear extrapolation procedure (dashed line) predicts a bulk thermal conductivity that is within $10 \%$ of $k_{\mathrm{LD}}$.

For SW silicon at a temperature of $1000 \mathrm{~K}$ (not shown), a nonlinear trend is also found between $k_{\mathrm{LD}} / k_{\mathrm{LD}}(L)$ and $1 / L$. As temperature is increased, the phonon-phonon-scattering rates increase due to increased anharmonicity, resulting in a reduction in the mean-free paths. Thus, the difference between the mean-free paths and sample lengths also decreases, resulting in more accurate predictions of the linear extrapolation procedure at higher temperatures $\left(k_{\mathrm{LD}}^{e} / k_{\mathrm{LD}}\right.$ $=0.48$ at $T=500 \mathrm{~K}$ while $k_{\mathrm{LD}}^{e} / k_{\mathrm{LD}}=0.57$ at $T=1000 \mathrm{~K}$ ). This increase in accuracy is not as significant as that seen in the MD results $\left(k_{\mathrm{DM}}^{e} / k_{\mathrm{GK}}=0.40\right.$ at $T=500 \mathrm{~K}$ while $k_{\mathrm{DM}}^{e} / k_{\mathrm{GK}}=0.66$ at $\left.T=1000 \mathrm{~K}\right)$. As temperature increases, the fifth-order and higher-order anharmonic terms neglected in the lattice dynamics calculations become more important. Since MD simulations include these higher-order terms, the difference between the mean-free paths and sample lengths decreases at a faster rate than in a lattice dynamics calculation as temperature increases.

\section{Discussion}

Previous applications of the direct method assumed that $1 / k$ and $1 / L$ are linearly dependent. If this assumption is applied to the discrete data points in Fig. 4(a), which correspond to lengths that span less than one order of magnitude $(40-80 \mathrm{~nm})$, one would conclude that the linear fit reasonably describes the $1 / k$ dependence on $1 / L$ and that a linear extrapolation procedure can be used to predict the bulk thermal conductivity. By analyzing results for sample lengths 
that span multiple orders of magnitude $\left(10-10^{7} \mathrm{~nm}\right.$, see Fig. 6), however, it becomes apparent that the observed linear relationship is a construct of using too small a range of sample lengths (i.e., any nonlinear function will appear linear if a small enough region is analyzed). Since the discrete data points in Fig. 4(a) are consistent with the range of sample sizes that are typically used in direct method MD simulations, it is not surprising that previous studies consistently observed a linear trend. . $^{5,10,16-22}$ For example, using samples lengths ranging from 29 to $128 \mathrm{~nm}$, Zhou et al. ${ }^{8}$ found that a linear relationship reasonably captures the $1 / k$ dependence on $1 / L$ for SW GaN at $T=300 \mathrm{~K}$. But at a temperature of $800 \mathrm{~K}$, where the phonon mean-free paths are reduced, they observed a nonlinear trend similar to that shown in Fig. 4(a). To explain this observation, they suggest that their direct method data at $T=800 \mathrm{~K}$ correspond to a nonlinear-transport regime (i.e., a breakdown of the Fourier law). Based on the results presented in Sec. III B for SW silicon, however, we believe that the nonlinear trend observed at $T=800 \mathrm{~K}$ is the true $1 / k$ dependence on $1 / L$, and that the linear trend found at $300 \mathrm{~K}$ is a result of using too small a range of sample lengths. Zhou et al. state that their observed nonlinear behavior calls into question the linear extrapolation procedure, a statement that is supported by this work.

From the results presented here, it is clear that caution should be used when attempting to predict bulk thermal conductivities using the linear extrapolation procedure. In Sec. III B, we showed that knowledge of the mode-dependent phonon mean-free paths is required to predict what sample lengths need to be considered for the linear extrapolation procedure to be appropriate. In practice, however, the linear extrapolation procedure will be applied to results from direct method MD simulations, where the phonon mean-free paths are not predicted. A more practical metric for determining when the linear extrapolation procedure is appropriate is thus needed.

\section{LENGTH-DEPENDENT THERMAL CONDUCTIVITY MODEL}

We now develop an analytical model for the length dependence of thermal conductivity. We will use this model to develop a metric to check if the sample sizes considered in direct method MD simulations are sufficiently large to accurately predict the bulk thermal conductivity using the linear extrapolation procedure.

We begin by converting the summation over all phonon modes in Eq. (3) to an integral over the first BZ, resulting in

$$
k(L)=\frac{V}{8 \pi^{3}} \sum_{\nu} \int c_{p h} v_{g, z}^{2} \tau d \boldsymbol{\kappa},
$$

where the relaxation time is length dependent. Assuming that optical phonons do not contribute to thermal conductivity and transforming the integral to spherical coordinates gives

$$
\begin{aligned}
k(L)= & \frac{V}{8 \pi^{3}} \sum_{\nu}^{a c} \int_{0}^{\kappa_{\mathrm{BZ}}(\theta, \phi)} \int_{0}^{2 \pi} \int_{0}^{\pi} c_{p h}\left|\mathbf{v}_{g} \cos \theta\right|^{2} \\
& \times \tau \kappa^{2} \sin \theta d \theta d \phi d \kappa,
\end{aligned}
$$

where $\kappa_{\mathrm{BZ}}(\theta, \phi)$ is the magnitude of the wave vector at the first BZ boundary along the $\theta$ and $\phi$ directions and the summation is restricted to acoustic modes $(a c)$. Note that multiplying the magnitude of the velocity vector by $\cos \theta$ gives its component in the [001] direction.

We now make the Debye approximation for the phonon dispersion, which assumes a single acoustic branch (i.e., no distinction between longitudinal and transverse polarizations), such that $\omega=v_{a c} \kappa$. Under this approximation for a classical system, Eq. (10) can be simplified to

$$
k(L)=\frac{3}{8 \pi^{3}} \int_{0}^{\omega_{\mathrm{D}}} \int_{0}^{2 \pi} \int_{0}^{\pi} k_{\mathrm{B}} \frac{\cos ^{2} \theta}{v_{a c}} \tau \omega^{2} \sin \theta d \theta d \phi d \omega,
$$

where the integral over wave vector has been changed to an integral over frequency and $c_{p h}$ has been replaced with $k_{\mathrm{B}} / V$. The Debye frequency, $\omega_{\mathrm{D}}$, is defined as ${ }^{26,27}$

$$
\omega_{\mathrm{D}}=v_{a c}\left(\frac{6 \pi^{2}}{\Omega}\right)^{1 / 3},
$$

where $\Omega$ is the volume of the primitive cell and is equal to $a^{3} / 4$ for face-centered cubic and diamond crystal lattices, where $a$ is the lattice constant.

The Matthiessen rule (see Sec. II B 2) is then used to combine the phonon-phonon and phonon-boundary scatterings so that

$$
\frac{1}{\tau}=\frac{1}{\tau_{\infty}}+\frac{2 v_{a c}|\cos \theta|}{L} .
$$

The phonon-phonon relaxation times are modeled using a relationship derived by Callaway for low frequencies, $\tau_{\infty}$ $=A / \omega^{2}$. This form is in agreement with the lattice dynamicspredicted phonon-phonon relaxation times for SW silicon at frequencies below $3 \mathrm{THz} .{ }^{30,31}$ The constant $A$ is calculated in the bulk limit and is

$$
A=\frac{2 \pi^{2} v_{a c} k_{\infty}}{k_{\mathrm{B}} \omega_{\mathrm{D}}}
$$

Substituting Eqs. (12)-(14) into Eq. (11) and integrating over $\theta, \phi$, and $\omega$ yields

$$
\begin{aligned}
k(\mathcal{L})= & k_{\infty}\left[\frac{6}{7}+\frac{3}{14} \mathcal{L}-\frac{3}{7} \mathcal{L}^{2}+\frac{3}{7} \mathcal{L}^{3} \ln \left(1+\frac{1}{\mathcal{L}}\right)\right. \\
& \left.-\frac{6}{7 \sqrt{\mathcal{L}}} \arctan (\sqrt{\mathcal{L}})\right],
\end{aligned}
$$

where $\mathcal{L}$ is a nondimensional length defined as 


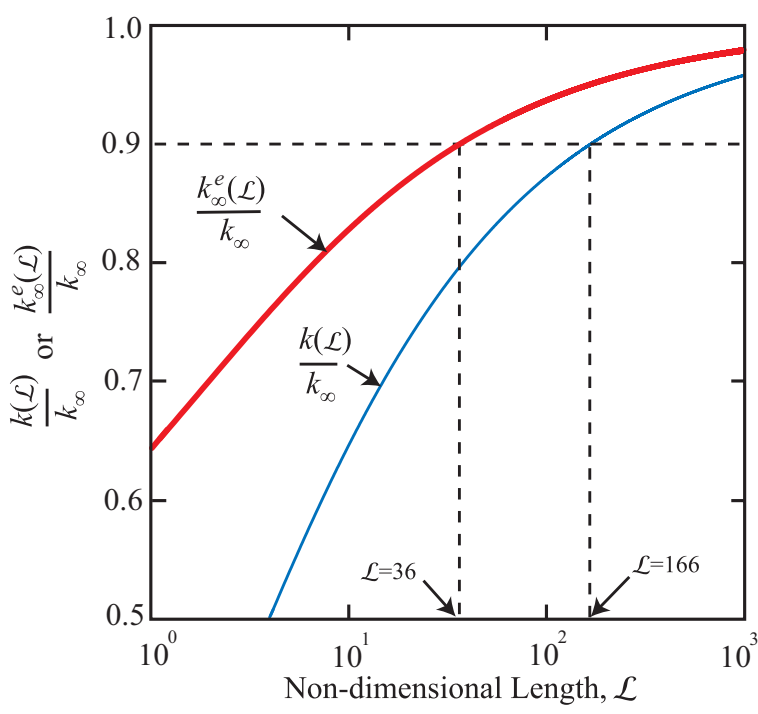

FIG. 7. (Color online) Length dependence of the thermal conductivity using Eq. (15) and the bulk thermal conductivity estimated using the linear extrapolation procedure [Eq. (17)].

$$
\mathcal{L} \equiv \frac{6 k_{\mathrm{B}} v_{a c} L}{k_{\infty} a^{3}}
$$

In the limit $\mathcal{L} \rightarrow \infty$, the bracketed term in Eq. (15) approaches unity and $k(\mathcal{L})$ approaches $k_{\infty}$. The assumptions underlying Eq. (15) are reasonable for single-element crystals with facecentered cubic or diamond lattices (e.g., argon and silicon). Optical modes contribute less than $5 \%$ to the bulk thermal conductivity of SW silicon ${ }^{30}$ and do not exist in crystals with a monoatomic primitive cell, such as argon.

Using Eq. (15) as a model for the length dependence of the thermal conductivity and the Taylor-series analysis introduced in Sec. II B 3, we now examine the linear extrapolation procedure. The bulk thermal conductivity estimated from the linear extrapolation procedure applied to a sample of nondimensional length $\mathcal{L}, k_{\infty}^{e}(\mathcal{L})$, is obtained by truncating the second-order and higher-order terms of the Taylor series in Eq. (8) such that

$$
k_{\infty}^{e}(\mathcal{L})=\frac{1}{\frac{1}{k(\mathcal{L})}+\frac{\partial\left(k^{-1}\right)}{\partial\left(\mathcal{L}^{-1}\right)}\left[-\frac{1}{\mathcal{L}}\right]} .
$$

The thermal conductivity predicted using Eq. $(15)[k(\mathcal{L})]$ and the bulk thermal conductivity estimated using the linear extrapolation procedure $\left[k_{\infty}^{e}(\mathcal{L})\right]$ are plotted in Fig. 7. Both thermal conductivities converge to the bulk value as the sample length increases. $k(\mathcal{L})$ is within $10 \%$ of the bulk value for a nondimensional length of 166 while a nondimensional length of 36 is required to obtain the same level of convergence when the linear extrapolation procedure is applied.

A nondimensional length of 36 corresponds to a dimensional- and material-specific length of

$$
L_{\min }=\frac{6 k_{\infty} a^{3}}{k_{\mathrm{B}} v_{a c}},
$$

where we define $L_{\min }$ to be the minimum sample length required to ensure that the bulk thermal conductivity predicted using the linear extrapolation procedure is within $10 \%$ of the true value. To evaluate $L_{\min }$, we take $v_{a c}$ to the be the average of the [001] acoustic phonon group velocities in the $\boldsymbol{\kappa} \rightarrow 0$ limit,

$$
v_{a c}=\frac{v_{a c, L}^{[001]}+2 v_{a c, T}^{[001]}}{3},
$$

where $L$ and $T$ indicate the longitudinal and transverse dispersion branches. Setting $k_{\infty}$ equal to $k_{\mathrm{LD}}$ and using the group velocities predicted from lattice dynamics calculations for SW silicon, $L_{\min }$ is $3.3 \mu \mathrm{m}(1.5 \mu \mathrm{m})$ at a temperature of 500 $\mathrm{K}(1000 \mathrm{~K})$. As shown in Fig. 6, this length is consistent with the sample lengths required to predict a bulk thermal conductivity that is within $10 \%$ of $k_{\mathrm{LD}}$ using the linear extrapolation procedure and lattice dynamics-predicted phonon properties in Eq. (6). Based on this result, we believe that Eq. (18) can be used to estimate the minimum sample length required when using the linear extrapolation procedure.

Since neither $v_{a c}$ nor $k_{\infty}$ is available prior to performing direct method MD simulations, Eq. (18) cannot be applied as is. We therefore substitute $v_{a c, L}^{[001]}=\left(C_{11} / \rho\right)^{1 / 2}$ and $v_{a c, T}^{[001]}$ $=\left(C_{44} / \rho\right)^{1 / 2}$ into Eq. (19), where $\rho, C_{11}$, and $C_{44}$ are the bulk material density and elastic constants. Substituting this definition for $v_{a c}$ into Eq. (18) and solving for $k_{\infty}$ results in

$$
k_{\infty}^{\max }=\frac{L k_{\mathrm{B}}\left(C_{11}^{1 / 2}+2 C_{44}^{1 / 2}\right)}{18 a^{3} \rho^{1 / 2}} .
$$

We define $k_{\infty}^{\max }$ to be the maximum thermal conductivity that can be accurately predicted using the linear extrapolation procedure with a minimum direct method sample length, $L$. In other words, if the bulk thermal conductivity estimated using the linear extrapolation procedure and direct method MD data $\left(k_{\mathrm{DM}}^{e}\right)$ exceeds $k_{\infty}^{\mathrm{max}}$, then the prediction is not accurate. The minimum sample length should be increased until $k_{\mathrm{DM}}^{e} \lesssim k_{\infty}^{\max }$.

Examining the minimum sample lengths used in our direct method MD simulations (26 nm for LJ argon and $40 \mathrm{~nm}$ for SW silicon) and using the material properties provided in Table III, $k_{\infty}^{\max }$ is $0.79 \mathrm{~W} / \mathrm{m} \mathrm{K}$ for $\mathrm{LJ}$ argon and $5.1 \mathrm{~W} / \mathrm{m} \mathrm{K}$ for SW silicon. This result indicates that using a minimum sample length of $26 \mathrm{~nm}$ for LJ argon is reasonable since $k_{\mathrm{DM}}^{e}=0.50 \mathrm{~W} / \mathrm{m} \mathrm{K}<k_{\infty}^{\mathrm{max}}=0.79 \mathrm{~W} / \mathrm{m} \mathrm{K}$. The agreement between $k_{\mathrm{DM}}^{e}$ and $k_{\mathrm{GK}}$ in Table II is therefore not surprising. For SW silicon, using a minimum sample length of $40 \mathrm{~nm}$ results in $k_{\mathrm{DM}}^{e}>k_{\infty}^{\max }$ for both temperatures tested (see Table II), indicating that larger sample sizes are required to achieve agreement between $k_{\mathrm{DM}}^{e}$ and $k_{\mathrm{GK}}$. This result is consistent with the findings presented in Sec. III B and those of Schelling et al., ${ }^{5}$ who report direct method results that are in better agreement with their Green-Kubo predictions using samples lengths up to $209 \mathrm{~nm}$ (for SW silicon at $1000 \mathrm{~K}$ ). 
TABLE III. Lattice constants, densities, and elastic constants for LJ argon and SW silicon at a temperature of $0 \mathrm{~K}$.

\begin{tabular}{lcc}
\hline \hline Property & LJ argon & SW silicon \\
\hline$a(\AA)$ & $5.269^{\mathrm{a}}$ & $5.431^{\mathrm{b}}$ \\
$\rho\left(\mathrm{kg} / \mathrm{m}^{3}\right)$ & $1813^{\mathrm{a}}$ & $2329^{\mathrm{b}}$ \\
$C_{11}(\mathrm{GPa})$ & $4.113^{\mathrm{c}}$ & $151.6^{\mathrm{d}}$ \\
$C_{44}(\mathrm{GPa})$ & $2.286^{\mathrm{c}}$ & $56.5^{\mathrm{d}}$ \\
\hline
\end{tabular}

aeference 23 .

${ }^{\mathrm{b}}$ Reference 32 .

'Reference 33.

${ }^{\mathrm{d}}$ Reference 34

\section{RECOMMENDATIONS}

As summarized in Sec. III C, care must be taken when applying the linear extrapolation procedure to predict bulk thermal conductivity using the results of direct method MD simulations. If systems sizes smaller than the largest bulk mean-free paths that dominate the thermal conductivity are considered, a linear relationship between $1 / k$ and $1 / L$ may be incorrectly inferred and the thermal conductivity can be severely underestimated. Based on the results presented in Secs. II and III and our previous experiences, $, 6,7,9,10,15$ we suggest the following procedure for using MD simulation to predict bulk thermal conductivity.

(1) Use the Green-Kubo method, which is discussed in Sec. II A. The Green-Kubo method is advantageous in that: (i) it uses equilibrium simulations, (ii) the full thermal conductivity tensor is predicted, and (iii) system-size effects can typically be eliminated with less than 10000 atoms. The disadvantage of the Green-Kubo method is that the converged value of the HCACF can be sometimes difficult, or impossible, to specify. Such behavior has been observed for disordered materials, ${ }^{6,11}$ superlattices, ${ }^{6,11}$ and molecular chains. $^{12-14}$

(2) If the converged value of the HCACF cannot be specified, use the direct method, which is described in Sec. II B. First, estimate $k_{\infty}^{\max }$ from Eq. (20). Then, perform direct method simulations using systems of increasing length until the thermal conductivity predicted from the linear extrapolation procedure, $k_{\mathrm{DM}}^{e} \simeq k_{\infty}^{\max }$.

(3) If the system lengths accessible in the MD simulations cannot generate $k_{\mathrm{DM}}^{e} \simeq k_{\infty}^{\max }$, then MD simulation cannot be used to predict the bulk thermal conductivity. In such a case, we recommend using phonon properties obtained from lattice dynamics calculations and Eq. (6) in the $L \rightarrow \infty$ limit. The technique we used to predict phonon properties, described in Sec. III, is based on harmonic and third-order anharmonic lattice dynamics calculations. Because higherorder terms in the potential energy are neglected, this approach is only expected to be valid up to about half the Debye temperature. ${ }^{7}$ An alternative method that includes the full anharmonicity of the atomic interactions is to calculate the phonon properties using the spectral energy density. We have described this approach elsewhere. ${ }^{35}$

\section{ACKNOWLEDGMENTS}

We acknowledge support from the Natural Sciences and Engineering Research Council of Canada Discovery Grants Program, the Pennsylvania Infrastructure Technology Alliance, and Advanced Micro Devices. We thank J. A. Thomas (Carnegie Mellon University) for providing suggestions to improve the manuscript.
*Corresponding author; mcgaughey@cmu.edu

${ }^{1}$ A. J. C. Ladd, B. Moran, and W. G. Hoover, Phys. Rev. B 34, 5058 (1986).

${ }^{2}$ D. A. McQuarrie, Statistical Mechanics (University Science Books, Sausalito, 2000).

${ }^{3}$ S. Berber, Y.-K. Kwon, and D. Tománek, Phys. Rev. Lett. 84, 4613 (2000).

${ }^{4}$ S. G. Volz and G. Chen, Phys. Rev. B 61, 2651 (2000).

${ }^{5}$ P. K. Schelling, S. R. Phillpot, and P. Keblinski, Phys. Rev. B 65, 144306 (2002).

${ }^{6}$ E. S. Landry, M. I. Hussein, and A. J. H. McGaughey, Phys. Rev. B 77, 184302 (2008).

${ }^{7}$ J. E. Turney, E. S. Landry, A. J. H. McGaughey, and C. H. Amon, Phys. Rev. B 79, 064301 (2009).

${ }^{8}$ X. W. Zhou, S. Aubry, R. E. Jones, A. Greenstein, and P. K. Schelling, Phys. Rev. B 79, 115201 (2009).

${ }^{9}$ A. J. H. McGaughey and M. Kaviany, Phys. Rev. B 69, 094303 (2004).

${ }^{10}$ E. S. Landry and A. J. H. McGaughey, Phys. Rev. B 79, 075316 (2009).

${ }^{11}$ E. S. Landry, Ph.D. thesis, Carnegie Mellon University, 2009.

${ }^{12}$ A. Henry and G. Chen, Phys. Rev. B 79, 144305 (2009).

${ }^{13}$ J.-S. Wang and B. Li, Phys. Rev. Lett. 92, 074302 (2004).

${ }^{14}$ J.-S. Wang and B. Li, Phys. Rev. E 70, 021204 (2004).
${ }^{15}$ A. J. H. McGaughey, M. I. Hussein, E. S. Landry, M. Kaviany, and G. M. Hulbert, Phys. Rev. B 74, 104304 (2006).

${ }^{16}$ A. J. H. McGaughey and M. Kaviany, in Advances in Heat Transfer, edited by G. A. Greene, Y. I. Cho, J. P. Hartnett, and A. Bar-Cohen (Elsevier, New York, 2006), Vol. 39, pp. 169-255.

${ }^{17}$ D. G. Cahill, W. K. Ford, K. E. Goodson, G. D. Mahan, A. Mujumdar, H. J. Maris, R. Merlin, and S. R. Phillpot, J. Appl. Phys. 93, 793 (2003).

${ }^{18}$ B. Ni, T. Watanabe, and S. R. Phillpot, J. Phys.: Condens. Matter 21, 084219 (2009).

${ }^{19}$ L. Shi, D. Yao, G. Zhang, and B. Li, Appl. Phys. Lett. 95, 063102 (2009).

${ }^{20}$ S.-C. Wang, X.-G. Liang, X.-H. Xu, and T. Ohara, J. Appl. Phys. 105, 014316 (2009)

${ }^{21}$ N. Papanikolaou, J. Phys.: Condens. Matter 20, 135201 (2008).

${ }^{22}$ J. A. Thomas, R. M. Iutzi, and A. J. H. McGaughey, Phys. Rev. B 81, 045413 (2010).

${ }^{23}$ A. J. H. McGaughey, Ph.D. thesis, University of Michigan, 2004.

${ }^{24}$ J. E. Turney, A. J. H. McGaughey, and C. H. Amon, Phys. Rev. B 79, 224305 (2009).

${ }^{25}$ J. V. Goicochea, M. Madrid and C. H. Amon, J. Heat Transfer 132, 012401 (2010).

${ }^{26}$ J. M. Ziman, Electrons and Phonons (Oxford University Press, 
New York, 2001)

${ }^{27}$ G. P. Srivastava, The Physics of Phonons (Adam Hilger, Bristol, 1990).

${ }^{28}$ M. T. Dove, Introduction to Lattice Dynamics (Cambridge University Press, Cambridge, 1993).

${ }^{29}$ A. A. Maradudin and A. E. Fein, Phys. Rev. 128, 2589 (1962).

${ }^{30}$ D. P. Sellan, J. E. Turney, A. J. H. McGaughey, and C. H. Amon (unpublished).
${ }^{31}$ E. S. Landry and A. J. H. McGaughey, Phys. Rev. B 80, 165304 (2009).

${ }^{32}$ F. H. Stillinger and T. A. Weber, Phys. Rev. B 31, 5262 (1985).

${ }^{33}$ D. J. Quesnel, D. S. Rimai, and L. P. DeMejo, Phys. Rev. B 48, 6795 (1993).

${ }^{34}$ E. R. Cowley, Phys. Rev. Lett. 60, 2379 (1988).

${ }^{35}$ J. A. Thomas, J. E. Turney, R. M. Iutzi, C. H. Amon, and A. J. H. McGaughey, Phys. Rev. B 81, 081411(R) (2010). 